\title{
Algebraic Specification of a Model Transformation Engine*
}

\author{
Artur Boronat, José Á. Carsí, and Isidro Ramos \\ Department of Information Systems and Computation, \\ Technical University of Valencia, \\ Camí de Vera s/n, \\ Valencia 46022, Spain \\ \{aboronat, pcarsi, iramos\}@dsic.upv.es
}

\begin{abstract}
In Model-Driven Engineering, a software development process is a sequence of manipulation tasks that are applied to models, where model transformations play a relevant role. MOMENT (MOdel manageMENT) is a framework that is integrated in the Eclipse platform. MOMENT provides a collection of generic set-oriented operators to manipulate EMF models. In this paper, we present the model transformation mechanism that is embodied by the ModelGen operator. This operator uses the term rewriting system Maude as transformation engine and provides support for traceability. ModelGen has been defined in an algebraic specification so that we can use formal tools to reason about transformation features, such as termination and confluence. Furthermore, its application to EMF models shows that formal methods can be applied to industrial modeling tools in an efficient way. Finally, we indicate how the ModelGen operator provides support for the QVT Relations language in the MOMENT Framework.
\end{abstract}

Keywords: Model-Driven Engineering, Model Transformation, QVT, Algebraic Specifications, Traceability.

\section{Introduction}

Nowadays, the Model-Driven Architecture (MDA) [1] and the Software Factories [2] initiatives are leading the Model-Driven Engineering field. Both agree that any software artifact in a software development process can be dealt with as a model. Models provide a more abstract description of a software artifact than the final code of the application. Therefore, working on models increases the productivity in a software development process. It also increases the portability and the quality of the final code by applying generative techniques. In MDA, model transformations have become a relevant issue by means of the forthcoming standard Query/Views/ Transformations (QVT) [3]. Since any software artifact can be viewed as a model, model transformation is the basic mechanism that permits the manipulation of software artifacts [4].

* This work was supported by the Spanish Government under the National Program for Research, Development and Innovation, DYNAMICA Project TIC 2003-07804-C05-01.

L. Baresi and R. Heckel (Eds.): FASE 2006, LNCS 3922, pp. $262-277,2006$.

(C) Springer-Verlag Berlin Heidelberg 2006 
Within this arena, the Model Management discipline [5] considers models as firstclass citizens and provides a set of generic operators to manipulate them: Merge, Diff, ModelGen, etc. We have developed a framework, called MOMENT (MOdel manageMENT) [6], that is embedded in the Eclipse platform and that provides a set of generic operators to deal with models through the Eclipse Modeling Framework (EMF). An algebra of model management operators has been specified generically by using the Maude algebraic specification formalism [7] in the MOMENT framework.

In this paper, we focus on the ModelGen operator, the model transformation mechanism of MOMENT, which was presented as a proposal in [8]. This operator provides support for the QVT Relations language [3] and also provides support for traceability. ModelGen is used by the other model management operators of the Framework when a model manipulation has to be performed. Since the ModelGen operator is algebraically specified in Maude, this term rewriting system is used as the underlying runtime environment for model transformations in MOMENT. This fact provides an efficient environment to execute the ModelGen operator and a formal environment where algebraic features can be proved, such as the confluence and the termination of a model transformation.

The structure of the paper is as follows: Section 2 presents an overview of the QVT support in the MOMENT Framework and an example; Section 3 presents the algebraic specification of the ModelGen operator, how it is related to a QVT transformation, and the execution of a model transformation in the Framework; Section 4 compares our approach with other model transformation tools. Finally, Section 5 summarizes the main contributions of this paper.

\section{Model Transformations in the MOMENT Framework}

The ModelGen operator embodies the model transformation mechanism in the MOMENT Framework. This operator has been algebraically specified, although we use it to manipulate graphical models. To deal with models from an industrial standpoint and to manipulate them from a mathematical standpoint, we use two complementary Technical Spaces. A Technical Space (TS) is a working context with a set of concepts, a body of knowledge, tools, required skills, and possibilities [9]. We use the EMF and Maude technical spaces in our Framework. The former is characterized by its interoperability with industrial tools for solving actual Software Engineering problems. The latter constitutes the formal backbone of our model management approach.

Maude is a declarative language in the strict sense of the word. That is, a Maude program is a logical theory, and a Maude computation is logical deduction using the axioms specified in the theory/program. Maude is based on rewriting logic, which includes membership equational logic [19]. In Maude, membership equational theories are defined in functional modules. Computation is the form of equational deduction in which equations are used from left to right as simplification rules, with the rules being Church-Rosser and terminating.

Each Maude module specifies not just a theory, but also an intended mathematical model. For functional modules such models consist of certain sets of data and certain functions defined on such data, and are called algebras. Under Church-Rosser and 
termination assumptions, the equations of a functional module evaluate algebraic expressions to a single final result. By definition, the results of operations in this algebra are exactly those given by the Maude interpreter. Thus, a Maude module can simultaneously be viewed as an executable formal specification and as a program.

To achieve the interoperability between the EMF and the Maude technical spaces, two kinds of bridges are used in our Framework: two for regular metamodels and one for the QVT Relations metamodel.

For regular metamodels, two bridges are defined between the two technical spaces, at the M2-layer and at the M1-layer (using the Meta-Object Facility [10] terminology). Both of these permit the integration of MOMENT with EMF. The projection mechanism at the M2-layer automatically obtains the algebraic specification $^{1}$ that corresponds to a regular metamodel, by applying generative programming techniques ${ }^{2}$. An algebraic specification that is generated in this way provides the constructors that are needed to define models of the corresponding metamodel as sets. The inverse projection mechanism that obtains an EMF metamodel from an algebraic specification is not relevant in our tool because the algebraic specification must conform to several features in order to be used by our operators and they should be automatically achieved. We also think that visual modeling environments are more suitable for defining these metamodels. At the M1layer, we have developed a bidirectional projection mechanism that permits us to project an EMF model as a term of an algebra and to project a term of a metamodel algebra as an EMF model. In this case, bidirectionality is needed to apply an operator to an input model since the input model must be serialized as a term and the output term must be deserialized into an EMF model in order to be persisted.

Another bridge is defined for the QVT Relations metamodel. This permits the projection of a QVT transformation as an algebraic specification. This specification constitutes the axiomatic presentation of the ModelGen operator, when a transformation is defined among several metamodels.

In Fig. 1, the projection bridges between the two Technical Spaces (EMF and Maude) that are used in this work are shown. On the one hand, the dashed arrows represent the projection mechanism that obtains an algebraic specification for a regular EMF metamodel. On the other hand, the dotted arrow represents the projection mechanism that obtains the algebraic specification of a model transformation between several metamodels, when it is invoked in one direction. The importation of metamodels in an EMF QVT transformation model is also projected into the Maude TS by using the Maude module importation mechanism (shown as continuous arrows in the figure). Taking into account both kinds of projectors, we can deal with a QVT Relations model as the description of a model transformation or as a

${ }^{1}$ The algebraic specification that is generated for a given metamodel (defined in EMF as an Ecore model) permits the representation of models as algebraic terms. Thus, models can be manipulated by our model management operators. Algebraic specifications of this kind do not specify operational semantics for the concepts of the metamodel; they only permit the representation of information for model management issues.

2 These bridges can also be formalized in MOMENT by considering the Maude metamodel. Then, the bridges can be defined as model transformations. 


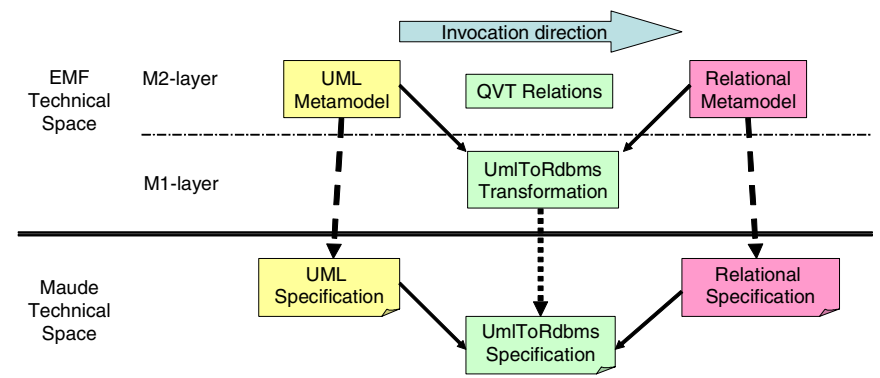

Fig. 1. Overview of the QVT support in the MOMENT Framework

regular model. Therefore, it can be transformed by using another QVT Relations model. This fact permits us to define the ModelGen operator as a mechanism to obtain higher order transformations through the QVT Relations language.

We have chosen the UmlToRdbms transformation that is presented in the QVT final adopted specification [3] as an example to illustrate the use of the ModelGen operator in the MOMENT Framework ${ }^{3}$. The Ecore metamodel [11] has been used as implementation of the UML metamodel. The RelationalDMBS metamodel of the QVT proposal has been specified as an EMF metamodel. Using both metamodels, the UmlToRdbms transformation is applied to the source UML model in Fig. 2 to obtain the target relational schema, which is shown in the figure by using the default EMF graphical modeller.

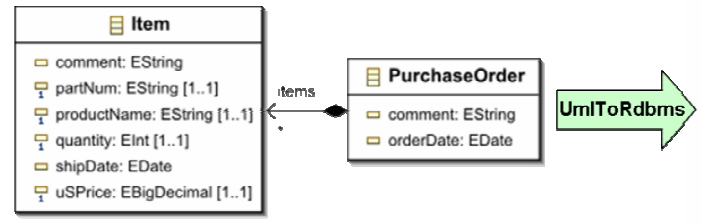

UML source model

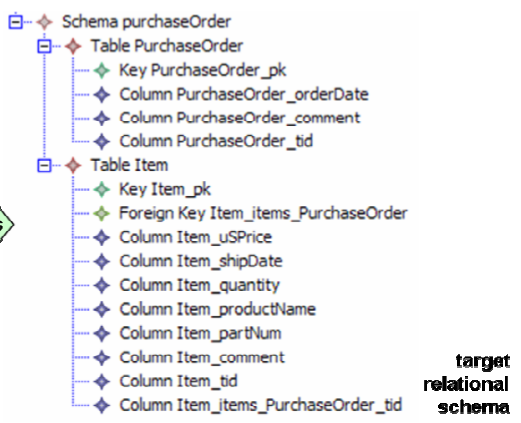

Fig. 2. Example of transformation of a UML model into a relational schema

\section{The Model Transformation Mechanism in MOMENT: The ModelGen Operator}

In this section, an overview of the QVT Relations language support in MOMENT that is based on the ModelGen operator is provided. We indicate how the model transformation in Section 2 is specified with the ModelGen operator, taking the Relations language as reference point.

\footnotetext{
${ }^{3}$ The complete algebraic specification of the example can be found at [25].
} 


\subsection{Overview of the Model Transformation Mechanism}

In the QVT Relations language, a model transformation is defined among several metamodels, which are called the domains of the transformation. A QVT transformation is constituted by QVT relations, which become declarative transformation rules. A QVT relation specifies a relationship that must hold between the model elements of different candidate models. The direction of the transformation is defined when it is invoked by choosing a specific domain as target. If the target domain is defined in the QVT transformation as enforceable, a transformation is performed. If the target domain is defined as checkonly, just a checking is performed.

In MOMENT, a QVT transformation is defined by means of the ModelGen operator. QVT relations are defined by means of the ModelGenRule operator ${ }^{4}$, which is used by the former operator. A model transformation can be applied to several source models, which may or may not conform to the same metamodel. It generates one target model and a set of traceability models. A traceability model contains a set of traces that relate the elements of the source model to the elements of the target model, indicating which transformation rule has been applied to each source element.

In this section, we present how MOMENT executes the ModelGen operator, transforming the UML model of the example in Section 2 into a relational schema. Fig. 3 shows the two MOF layers involved in a model transformation: the M2-layer, where the metamodels are defined; and the M1-layer, where the model transformation and the models are defined and manipulated. The front part of the figure represents the front-end of the MOMENT framework, i.e. EMF and all the plug-ins that are built on it. The back part of the figure represents the formal back-end of the MOMENT Framework, where Maude remains. The traceability support has not been taken into account in the figure.

Fig. 3 represents the transformation of the UML model by using the ModelGen operator. The steps that are automatically performed by the MOMENT Framework when the ModelGen operator is applied to the source UML model are the following:

- (1) and (2): We specify both UML and RelationalDMBS metamodels at the M2layer by means of the EMF or graphical editors based on this modelling framework. For instance, we can also consider XML schemas and Rational Rose models as metamodels.

- (3): The QVT transformation is defined as a model at the M1-layer, but it relates the constructs of the source to the constructs of the target metamodels. The transformation has to be defined as a model that conforms the QVT Relations metamodel by means of a graphical interface or as a program using the Relations language. The transformation model can either be defined by the user or automatically produced by another transformation.

- (4): We define a UML model using a UML graphical editor based on EMF.

- (5) and (6): Both UML and RelationalDBMS metamodels, respectively, are projected as algebraic specifications by means of the interoperability bridges that

${ }^{4}$ In the MOMENT framework, ModelGen and ModelGenRule axioms are just generated when the target domain of the invoked transformation is defined as enforceable. The algebraic support for checkings is provided by means of boolean operators and it is out of the scope of the paper. 
have been implemented in the MOMENT Framework. These algebraic specifications permit each metamodel to be considered as an algebra that provides the constructors to build models as algebraic sets.

- (7): The model that defines the QVT transformation is projected into the Maude TS as the UmlToRdbms algebraic specification, which contains the specification of the ModelGen and ModelGenRule operators.

- (8): The source UML model, which is defined in step (4) at the M1-layer, is projected into the Maude TS as a term of the UML algebra (9).

- (10): Maude applies the ModelGen operator through its equational deduction mechanism, obtaining a term of the RelationalDBMS algebra (11). Thus, Maude constitutes the runtime engine for the MOMENT transformation mechanism.

- (12): This is the last step of the model transformation process. It parses the term (11), defining an EMF model (13) in the M1-layer, which conforms to the target metamodel defined at the M2-layer.

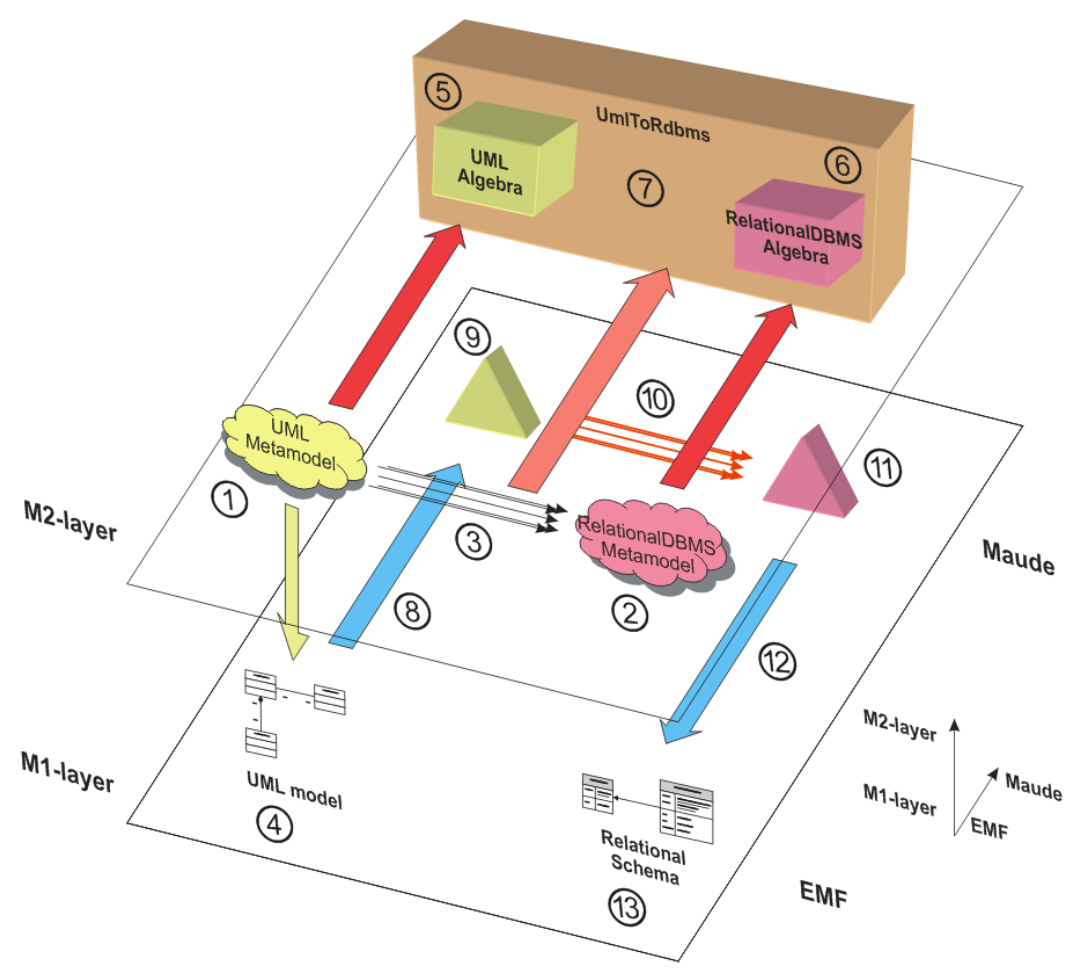

Fig. 3. Steps performed by the MOMENT model transformation mechanism

In the model transformation process, the user only interacts with the MOMENT framework when defining the source and target metamodels (step (1) and (2)), the QVT transformation between both metamodels (step (3)) and the source model (step (4)). The other steps are automatically carried out by the framework. The output model can also be manually manipulated from a graphical editor. In this paper, we 
focus on the steps (7) and (10), indicating the structure of the ModelGen operator and how it is related to the QVT Relations metamodel.

\subsection{Relations: The ModelGenRule Operator}

In MOMENT, a QVT transformation is projected into several directed transformations, by means of ModelGen operator specifications. There is exactly one transformation for each direction in which the QVT transformation can be invoked. QVT relations of a QVT transformation are projected as ModelGenRule axioms, once the transformation direction is determined. The operation ModelGenRule that permits the definition of directed transformation rules is declared as follows:

op ModelGenRule (_;_' : RelationSymbol ParameterList ->

Tuple\{ TTargetMM, TTraceabilityMetamodel,...\} .

The ModelGenRule operator has two parameters: the name of the relation (a term of the sort RelationSymbol) and a polymorphic list of parameters. The membership equational logic [7] is used to define the operational semantics of this operator by means of equations. To use the Maude equational deduction as the runtime engine for our transformation rules, we need them to be confluent and terminating. The first parameter makes the confluence satisfaction easier to achieve. It permits to differentiate two transformation rules, even though they have the same list of parameters. Therefore, we avoid the situation where several equations can be applied to reduce the same term. We discuss the termination issue in more depth in Section 3.2.2. The second parameter permits the definition of a polymorphic list of parameters for the transformation rule. This means that we can define a parameter of any type (either a model or a basic type) as input for the transformation rule.

The result of a transformation rule is a tuple of several elements, where an element can be a model conforming any of the metamodels involved in the transformation. Among the models that are produced by a transformation rule, we distinguish the target model and several traceability models. There is one traceability model for each pair (source model, target model).

\subsubsection{Structure of a Transformation Rule in MOMENT}

We can only know the direction of a transformation by means of the enforceable property during the invocation process. During this process, the ModelGenRule axioms that specify the operational semantics of the relation are generated to be invoked later. Fig. 4 shows the two parts that constitute a ModelGenRule axiom: the specification of the model transformation and the specification of the traceability model definition. The Maude code that is generated for each part of a QVT relation is structured as shown in Fig. 4.

- The Relation Symbol. This is the name of the transformation rule. It is used to define a constructor for the sort RelationSymbol, which is used in the ModelGenRule axioms for the sake of confluence.

- The Domains. They constitute the patterns of the transformation rules and the body, using constructs of the involved metamodels. Two kinds of domains can be distinguished in the transformation invocation: 
- The domain that is selected as target, when the transformation is invoked, is used to generate the body of the transformation rules. In the body of a transformation rule, new instances can be created in the target model and expressions to obtain the information from the source models can be used. Expressions of this kind are called object template expressions. In them, OCL is used as the query language and new functions can be declared in a transformation to manipulate data. On the one hand, a parametric algebraic specification has been defined to provide the operational semantics for OCL 2.0 expressions $^{5}$ in MOMENT. On the other hand, Maude itself is suitable to define the operational semantics of QVT functions.

- The source domains of the QVT relation are used to define the pattern of the transformation rule. In the pattern, each input model appears twice. This is needed to achieve two goals at the same time: to use the pattern matching mechanism that Maude provides by means of recursion and to provide support for OCL expressions. The first model is used to search the corresponding element of the pattern that is needed in the transformation rule. The second model is needed because Maude does not provide support for side effects, as in pure functional programming languages. This forces us to keep the whole model throughout the term reduction process in order to navigate it by means of OCL expressions, which can be used in the guard of the pattern. In the following section, we study the pattern matching mechanism in further detail.

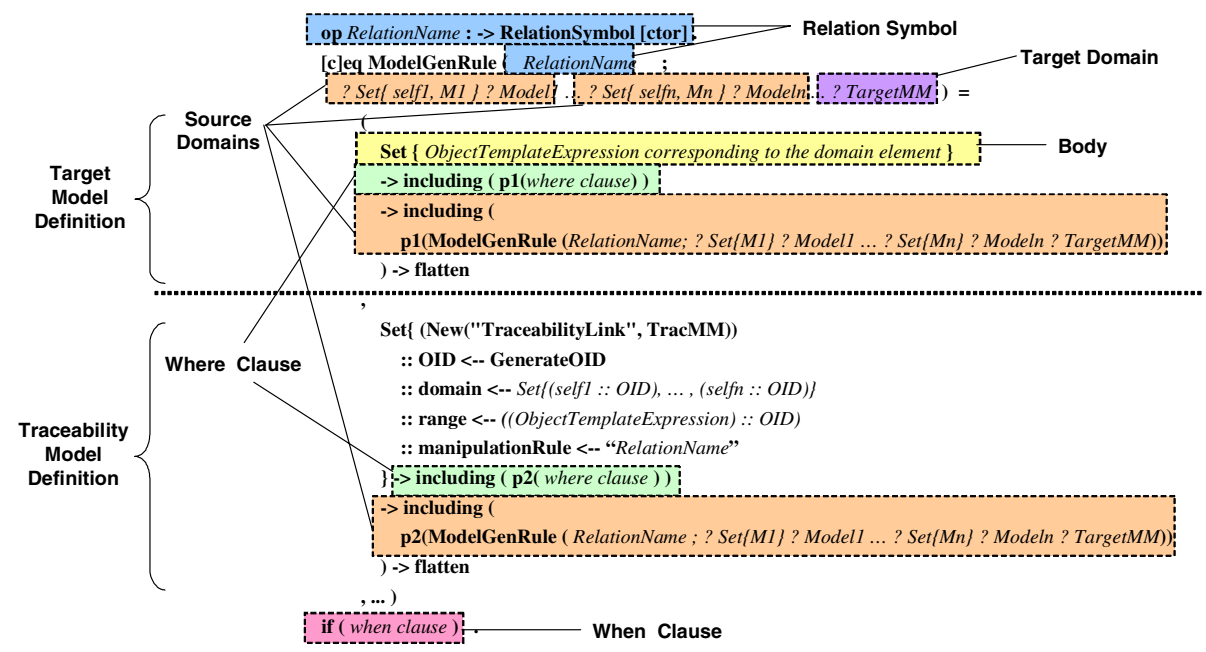

Fig. 4. Axiom for the ModelGenRule operator

- The When clause. This clause is used as a precondition for the transformation rule. Therefore, this guard participates in the pattern matching mechanism. It is used to obtain the condition of the equations that constitute the transformation rule. The

${ }^{5}$ The OCL 2.0 algebraic support is out of the scope of this paper. 
resulting equations are applied by the conditional pattern matching mechanism of Maude.

- The Where clause: this clause is used as postcondition for a transformation rule. In MOMENT, it is used to generate the code that invokes other QVT relations. In this clause, variables can also be initialized with new values to be used in the transformation.

In the QVT proposal, the traceability support is implicit in the QVT Relations language and is explicit in the QVT Core language. The ModelGen operator also generates traceability models automatically, but its definition has to be explicitly specified in the ModelGenRule axioms. The traceability model produced by the ModelGen operator conforms to the traceability metamodel that is presented in [12]. Thus, it can also be manipulated as just another model by the operator ModelGen.

In the second part of the axiom shown in Fig. 4, a new trace (instance of the TraceabilityLink class) is added to the traceability metamodel. Its domain field is filled with the identifiers (or references) of the source elements that have been matched with the pattern. Its range field is filled with the identifiers of all the instances that are created in the body of the transformation rule. Finally, the manipulationRule field indicates which transformation rule has been applied. Since the axioms for the ModelGenRule transformation rules are automatically generated from a QVT relations model, the traceability support in MOMENT is automatically generated and is kept hidden from the final user.

As an example of a ModelGenRule axiom, we show the axiom that represents the regular case pattern for the following relation PackageToSchema of the UmlToRdbms transformation (when the transformation is applied to a UML model):

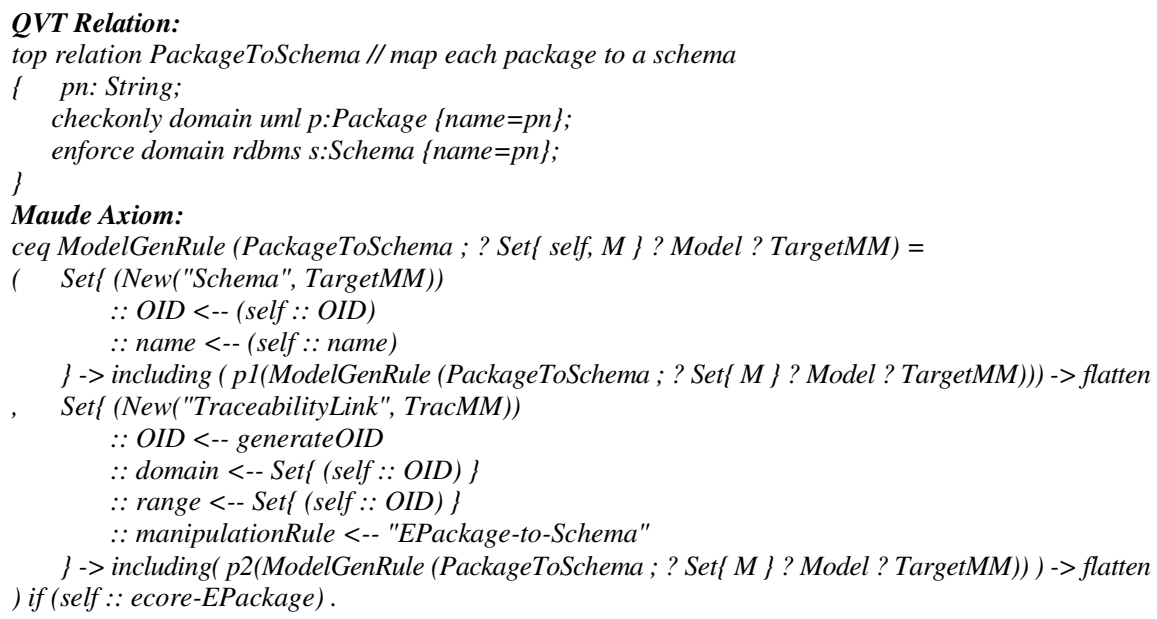

\subsubsection{Pattern Matching}

The pattern matching mechanism that is used to apply the transformation rules to source models is provided by Maude. To understand how the ModelGenRule uses it, we study the parts of a transformation rule that are involved in the pattern matching: the source domains and the when clause. 
A source domain is a model used as input in a transformation. It is a set that is constituted by an associative commutative magma ${ }^{6}$ of elements. The generic constructors for a Set are the following:

$$
\begin{aligned}
& o p_{\lrcorner_{-}}: \operatorname{Magma}\{X\} \operatorname{Magma}\{X\}->\operatorname{Magma}\{X\} \text { [assoc comm ctor] . } \\
& \text { op Set }\{\} \text { : Magma }\{X\}->\operatorname{Set}\{X\}[\text { ctor] . }
\end{aligned}
$$

Taking into account that the sort that represents model elements is a subsort of $\operatorname{Magma}\{X\}$, a UML model that is constituted by a package and a class can be defined as a set with the following term: Set\{(UML-Package ...), (UML-Class ...) \}; where $U M L-P a c k a g e$ and UML-Class are the constructors that correspond to these concepts in the Maude projection of the UML metamodel. By using a recursion mechanism we can use the Maude pattern matching mechanism. We define the following variables: the variable $\operatorname{self}_{i}$ can be bound to a term that represents any element of a Model $_{i}$ model. The variable $M_{i}$ can be bound to any magma of elements of a Model $_{i}$ model. For each QVT relation, we obtain two axioms for the ModelGenRule operator by using the following patterns:

- The base case Set\{ self $f_{i}$ : the right-hand side of this equation is constituted by the tuple of the target model and the traceability models.

eq ModelGenRule $\left(\right.$ RelationName ; ? Set $\left\{\operatorname{self}_{i}\right\} ?$ Model $_{i} \ldots$ ? TargetMM $)=(\operatorname{Set}\{\ldots\}, \operatorname{Set}\{\ldots\}, \ldots)$.

- The regular case $\operatorname{Set}\left\{\operatorname{self}_{i}, M_{i}\right\}$ : the right-hand side of this equation is constituted by the tuple of the target model and the traceability models, but also contains the application of the recursive transformation rule RelationName to the models that are constituted by the residuary magmas of elements. In the first element of the tuple, we apply the recursive operation and we add the first argument of the returning tuple by means of $p 1$. In the second element, we use $p 2$ to get the second element of the returning tuple.

eq ModelGenRule (RelationName ; ? Set\{ self $\left._{i}, M_{i}\right\}$ ? Model ... ? TargetMM) =

(Set\{...\}->including(p1(ModelGenRule (PackageToSchema ; ? Set\{ $\left.M_{i}\right\}$ ? Model ${ }_{i}$ ? TargetMM)))->flatten

Set $\{. .$.$\} ->including(p2(ModelGenRule(PackageToSchema ; ? Set \left\{M_{i}\right\}$ ? Model $_{i}$ ? TargetMM)) )->flatten).

The when clause also provides relevant information to determine whether or not an axiom can be matched to perform a transformation. When the guard described in the when clause does not contain an OCL query that searches elements through a model, it can be added to the axiom as a condition, turning the axiom into a conditional equation. When the guard contains an OCL query, the guard is added as an if...then...else...fi clause in the body of the equation. Both considerations are needed for the sake of the efficiency of the Maude AC matching algorithm.

When no axiom of the ModelGenRule operator can be applied, there is a general axiom for all the transformation rules that is applied by default. This axiom guarantees the termination of a transformation and is specified as follows:

$$
\begin{aligned}
& \text { var TR : RelationSymbol } . \quad \text { var PL }: \text { ParameterList } . \\
& \text { eq ModelGenRule }(T R ; P L)=(\text { empty-set } \text { empty-set })[\text { owise }] .
\end{aligned}
$$

\footnotetext{
${ }^{6} \mathrm{~A}$ magma of elements represents a group of elements that may be encapsulated in a set.
} 


\subsection{Transformations: The ModelGen Operator}

The ModelGen operator provides the model transformation mechanism in the MOMENT Framework. This operator corresponds to a QVT transformation when it is invoked in a specific direction. The ModelGen operator is declared as follows:

op ModelGen (_;_') : TransformationSymbol ParameterList

$\rightarrow$ Tuple\{ TTargetMM, TTraceabilityMetamodel,... \} .

Similarly to the ModelGenRule operator, it has two formal parameters: the symbol that represents the name of the transformation and a polymorphic list of parameters for the transformation. The result of the operator is a tuple that is constituted by the resulting target model and by traceability models. There is one traceability model for each pair (source model, target model).

The code that is generated for a transformation includes the definition of the symbol of the transformation and an axiom that specifies the semantics of the transformation. Fig. 5 shows the structure of an axiom for the ModelGen operator. With regard to the definition of a QVT transformation, we identify the following parts:

- The transformation symbol: represents the name of the transformation.

- The parameters of the transformation:

- Source Domains. ? SourceModel1 ... ? SourceModeln constitute a list of terms that represent the source models of the transformation.

- Target Domain. TargetMM is a term that represents the target metamodel. It is used to create new instances of the classes that appear in the metamodel by means of the Maude reflection mechanism.

- List of names of the input and target models. This list is used to define traceability models. This information is needed to indicate the models that are related by means of a traceability model.

- Target model definition. This is the first argument of the resulting tuple, where the target model is generated by applying the ModelGenRule axioms to the elements of the source models.

- Traceability model definition. The other arguments of the resulting tuple are the traceability models that relate the elements of the target model to those of each source model. Furthermore, a new instance of the TraceabilityModel class is created to relate a source model to the target one.

The operator CompleteReferences is needed to simplify the definition of transformation rules when the metamodel has opposite references. In EMF, UML associations that may appear in a MOF metamodel are defined by means of opposite references (two instances of the EReference class) [11]. If the metamodel has opposite references, both references must be initialized when a model is defined. This fact can make the definition of transformations more complex. Although the EMF editor solves this problem automatically, an independent solution is needed. The CompleteReferences operator fulfils this goal. Thus, it permits MOMENT to remain independent of any other technological space that is different from the Maude TS and to compose several transformations without loss of information. This operator has two 


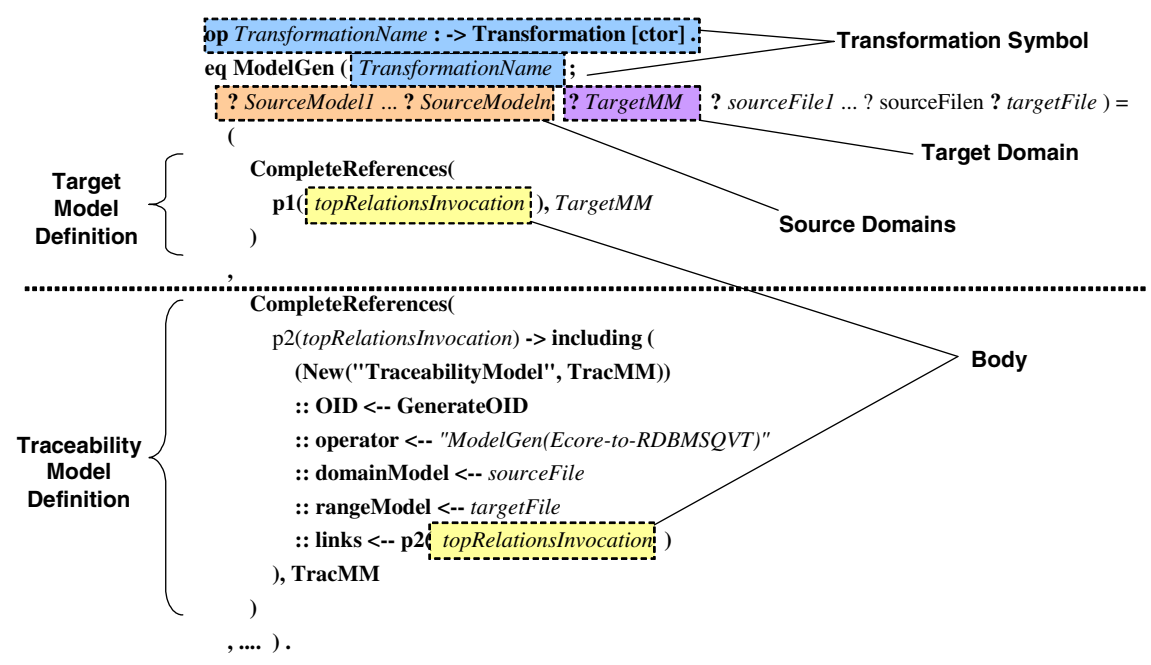

Fig. 5. Axiom for the ModelGen operator

input parameters: the model to be completed with references and the corresponding metamodel, which is expressed as a model conforming the Ecore metamodel. The CompleteReferences operator uses the Maude reflection mechanism to traverse the whole input model in order to complete the forgotten references. The output of the operator is the completed model.

Taking into account the example of the UmlToRdbms transformation that is presented in the MOF QVT final specification, we indicate the Maude code that provides the semantics of the transformation when it is invoked to transform a UML model into a relational schema. In the ModelGen axiom, we have only added the code to obtain the first element of the returning tuple, i.e. the target model.

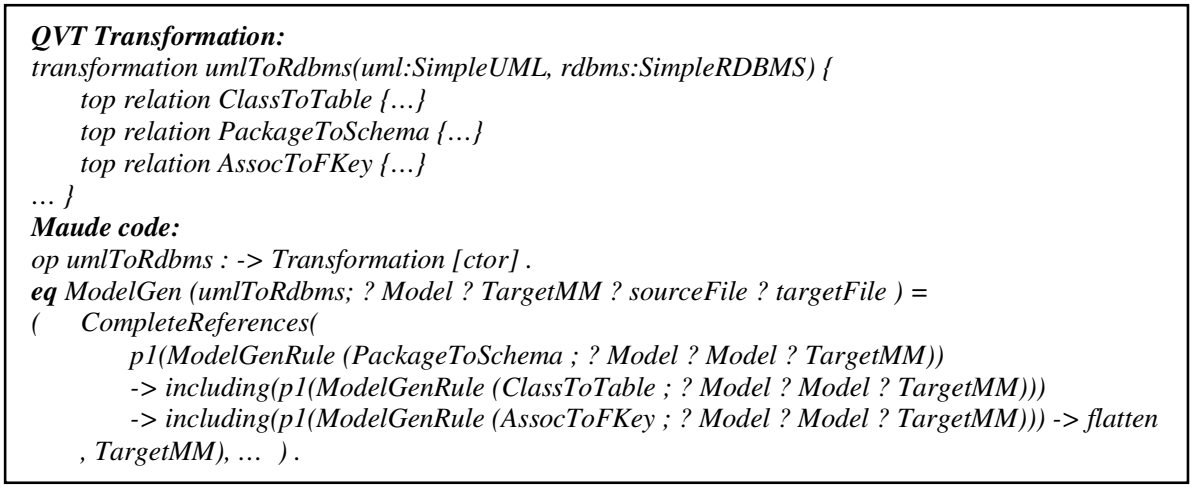

\section{Related Work}

We provide a brief comparison of the ModelGen operator to other model transformation mechanisms that are the most current in the Model-Driven Engineering field [20]. 
The goal of the study is to compare their support for traceability and transformation organization.

MTF [13] is the IBM Model Transformation Framework, which implements some of the QVT concepts and is based on the EMF. It provides a simple declarative language for defining mappings between models. An MTF transformation results in a set of mappings that relate objects among different models. The direction of the transformation is defined when the transformation is invoked. Bidirectional transformations imply constraining the kind of model transformations that can be solved. For instance, transformations that produce loss of information cannot be taken into account.

ATL [14] is a model transformation language that provides declarative and imperative constructs. A transformation is constituted by several transformation rules but there is no mechanism to organize them by means of modules. Traceability support is not provided implicitly in a transformation; traceability models can be generated by a transformation as in the ModelGen operator. The expressions in a transformation rule are defined in OCL expressions, making the ATL language easy to learn and to use. Tefkat [15] is a model transformation tool that is quite similar to ATL, which is also built on EMF. It incorporates the concept of tracking classes to define traces between the source model and the target model that is generated by the transformation. However, traces must be defined explicitly.

XSLT has become a popular alternative for describing model transformations. Tools, such as MTRANS [16] or UMT [17], follow this approach by serializing metamodels and models into XMI documents and then performing the transformations by means of XSLT specifications. Nevertheless, the verbosity of the XML syntax sometimes leads to specifications that are difficult to read and to maintain [18]. XSLT 2 permits the definition of transformations that generate more than one XML document. Therefore, traceability support can be added explicitly to a XSLT transformation. This approach can also be applied to EMF models, which are persisted in XMI. EMF avoids the serialization of certain data, such as derived attributes or default values. Thus, this functionality must be embedded in the XSLT specification. This task can easily become cumbersome.

In [21], graph transformation technology is studied by applying a taxonomy. Graph-based model transformations usually take advantage of the visual nature of the graphs to specify transformation rules. Composition of graph transformations can be achieved by using controlled or programmed graph transformation, such as sequencing, branching or looping. For instance, Fujaba [22] uses story diagrams for this purpose, while VIATRA [23] uses abstract state machines. In Fujaba, transformations are implemented as method bodies, so composition of transformations can be achieved by performing method calls. In MOMENT, transformations are defined in algebraic modules that can be imported into others. Furthermore, the ModelGen operator can be easily composed with other operators by using functional composition since we are working in an algebraic context. This feature avoids the development of complex frameworks to deal with transformation composition. Since the algebraic definition of the ModelGen operator is automatically generated from a QVT model, MOMENT can use standard-based notation, graphical or textual, to specify transformations.

Graph-based model transformation technology has a formal background where mathematical features such as confluence and termination can be proved. For 
instance, AGG provides the mechanism of critical pair analysis to check termination and confluence of graph grammars [24]. As a Maude module is by construction a mathematical object, we can directly use all the tools of mathematics and logic, including automatic or semiautomatic tools, to reason about the correctness of Maude modules. The Maude interpreter itself is the first and most obvious such tool. In fact it is a high-performance logical engine to prove logical facts about our theories. Furthermore, Maude has a collection of formal tools supporting different forms of logical reasoning to verify program properties that can be directly applied to the algebraic specifications obtained in MOMENT, including: an inductive theorem prover (ITP) to verify properties of functional modules; a Church-Rosser checker, to check such a property; a Knuth-Bendix completion tool and termination checker.

Among the studied tools, MTF is the only one that provides implicit traceability support. In the other approaches, the traceability support must be explicitly codified in the transformation function to generate a traceability model. In MOMENT, the set of axioms that describe the ModelGen operator is automatically generated from a QVT Relations model. Thus, the traceability support is also implicit.

\section{Conclusions}

In this paper, we have presented the ModelGen operator, which permits the definition of directed declarative transformations that can be applied to several source models, which may or may not conform to the same metamodel. Furthermore, a transformation can be parameterized with additional data that can act as control parameters allowing configuration and tuning. The return value of a transformation is a tuple that is constituted by a model and several traceability models. There is one traceability model for each pair (source model, target model). The traceability support that is provided in MOMENT [12] permits the definition of an incremental transformation operator in an easy way, similar to the PropagateChanges operator, which is also presented in [12].

Our formal approach for model transformation permits the application of advantageous features to this field, such as transformation composition or modularity. Furthermore, formal features (like confluence and termination) can also be studied. The MOMENT Framework shows that formal methods can be applied to industrial tools not only for proving theoretical aspects, but also for solving actual problems in an efficient manner. Nevertheless, an algebraic setting might not be the most userfriendly environment to work on models. This reason led us to provide support for QVT by using generative programming techniques. In this way, Maude remains hidden from the final user although its formal features are used in our framework.

\section{References}

1. Frankel, D. S.: Model Driven Architecture: Applying MDA to Enterprise Computing. John Wiley \& Sons OMG Press. January, 2003.

2. Greenfield, J., Short, K., Cook, S., Kent, S.: Software Factories: Assembling Applications with Patterns, Models, Frameworks, and Tools. John Wiley \& Sons. 2004. 
3. OMG, "MOF 2.0 QVT final adopted specification (ptc/05-11-01)", November 2005.

4. Sendall, S., Kozaczynski, W. Model Transformation: The Heart and Soul of Model-Driven Software Development. IEEE Software. Sep/Oct 2003 (Vol. 20, No. 5), pp. 42-45.

5. Bernstein, P.A: Applying Model Management to Classical Meta Data Problems. pp. 209220, CIDR 2003.

6. The MOMENT web site: http://moment.dsic.upv.es:8080

7. Clavel, M., Durán, F., Eker, S., Lincoln, P., Martí-Oliet, N., Meseguer, J., Quesada, J.F.: Maude: specification and programming in rewriting logic. Theoretical Computer Science, 285(2):187-243, 2002.

8. Boronat, A., Carsí, J.Á., Ramos, I.: Automatic Reengineering in MDA Using Rewriting Logic as Transformation Engine. IEEE Computer Society Press. 9th European Conference on Software Maintenance and Reengineering. Manchester, UK. 2005.

9. Kurtev, I., Bézivin, J., Aksit, M.: Technological Spaces: An Initial Appraisal. Int. Federated Conf. (DOA, ODBASE, CoopIS), Industrial track, Irvine, 2002.

10. OMG, "Meta Object Facility 1.4", http://www.omg.org/technology/documents/formal/ mof.htm

11. EMF web site: http://www.eclipse.org/emf/

12. Boronat, A., Carsí, J.Á., Ramos, I.: Automatic Support for Traceability in a Generic Model Management Framework. LNCS 3748. In Proceedings of European Conference on Model Driven Architecture - Foundations and Applications. Nuremberg (Germany). 2005.

13. The MTF web site: http://www.alphaworks.ibm.com/tech/mtf

14. Bézivin, J., Dupé, G., Jouault, F. , Pitette, G., and Rougui, E.J.: First experiments with the ATL model transformation language: Transforming XSLT into XQuery. In: OOPSLA 2003 Workshop, Anaheim, California.

15. Lawley, M., Steel, J.: Practical declarative model transformation with tefkat. In Model Transformations In Practice Workshop, Montego Bay, Jamaica, October 2005.

16. Peltier, M., Bézevin, J., Guillaume, G.: MTRANS: A general framework, based on XSLT for model transformations. In WTUML '01, Proceedings of the workshop on Transformations in UML, Genova, Italy, 2001.

17. The UMT web site: umt-qvt.sourceforge.net/

18. Gerber, A., Lawley, M., Raymond, K., Steel, J., Wood, A.: Transformation: The Missing Link of MDA, In A. Corradini, H. Ehrig, H.-J. Kreowski, G. Rozenberg (Eds.): Graph Transformation: First International Conference (ICGT 2002), v1 15 Barcelona, Spain, October 7-12, 2002. Proceedings. LNCS vol. 2505, Springer-Verlag, 2002, pp. 90 - 105

19. Meseguer, J.: Membership algebra as a logical framework for equational specification. In Francesco Parisi-Presicce, editor, Recent Trends in Algebraic Development Techniques, 12th International Workshop, WADT'97, Tarquinia, Italy, June 3-7, 1997, Selected Papers, volume 1376 of Lecture Notes in Computer Science, pages 18-61. Springer, 1998.

20. Czarnecki, K., Helsen, S.: Classification of Model Transformation Approaches. OOPSLA'03 Workshop on Generative Techniques in the Context of Model-Driven Architecture.

21. Mens, T., Van Gorp, P., Varro, F., Karsai, G.: Applying a model transformation taxonomy to graph transformation technology. Proc. Int'l Workshop on Graph and Model Transformation (GraMoT 2005). September 2005.

22. Burmester, S., Giese, H., Niere, J., Tichy, M., Wadsack, J., Wagner, R., Wendehals, L., Zuendorf, A.: Tool integration at the meta-model level: The fujaba approach. Int'l Journal on Software Tools for Technology Transfer 6 (2004), pp. 303-318. 
23. Csertán, G., Huszerl, G., Majzik, I., Pap, Z., Pataricza, A., Varró, D.: VIATRA - visual automated transformations for formal verification and validation of UML models, in: Proc. 17th Int'l Conf. Automated Software Engineering (2002), pp. 267-270.

24. Heckel, R., Küster, J., Taentzer, G.: Confluence of typed attributed graph transformation systems, in: Proc. 1st Int'l Conf. Graph Transformation, LNCS 2505 (2002), pp. 161-176.

25. Algebraic specification of the model transformation that is used as example in the paper: http://moment.dsic.upv.es/publications/fase06/example.html 